\title{
Increased incidence of invasive group A streptococcal disease in Ireland, 2012 to 2013
}

M Meehan (meehanmary@yahoo.co.uk)1, S Murchan², S Bergin³, D 0’Flanagan², R Cunney',2,3

1. Epidemiology and Molecular Biology Unit and, Irish Meningococcal and Meningitis Reference Laboratory, Temple Street Children's University Hospital, Dublin, Ireland

2. Health Protection Surveillance Centre, Dublin, Ireland

3. Department of Medical Microbiology, Temple Street Children’s University Hospital, Dublin, Ireland

Meehan M, Murchan S, Bergin S, O'Flanagan D, Cunney R. Increased incidence of invasive group A streptococcal disease in Ireland, 2012 to 2013. Euro Surveill. 2013;18(33):pii=20556. Available online: http://www.eurosurveillance.org/ViewArticle.aspx?Articleld=20556

Invasive group A streptococcal (iGAS) infections have been notifiable in Ireland since 2004. Incidence rates (2004-2011) have ranged from 0.8 to 1.65 per 100,000 . In 2012 , the iGAS rate rose to 2.66 per 100,000 and was associated with a high proportion of emm1 isolates. A further increase in January to June 2013 has been associated with increased prevalence of emm3. Public health departments and clinicians have been alerted to this increase.

In this communication, we report the increased incidence of severe Streptococcus pyogenes (group A streptococcus; GAS) infection in Ireland and a predominance of emm1 in 2012. In the first six months of 2013, we observed a further increase in iGAS and the emergence of emm3.

\section{Background}

S. pyogenes is a major human pathogen that causes a wide spectrum of clinical manifestations. These range from common superficial skin infections and pharyngitis to invasive infections such as bacteraemia, meningitis, cellulitis, pneumonia, and the more severe necrotising fasciitis (NF) and streptococcal toxic shock syndrome (STSS). Invasive GAS (iGAS) infections, though relatively uncommon compared to highly prevalent non-invasive GAS infections, remains a significant global cause of morbidity and mortality. Seven-day case fatality rates range from $8 \%$ to16\%, with high rates approaching $50 \%$ often associated with STSS [1].

The M protein, encoded by the emm gene, is the major virulence factor and an important epidemiological typing tool. In excess of $200 \mathrm{emm}$ types have been documented to date [2]. Whereas all types may be associated with invasive GAS (iGAS), emm1 and emm 3 have been particularly associated with severe iGAS infections and the resurgence of these infections that has occurred since the 1980 s [3]. The incidence of iGAS disease exhibits seasonal patterns in addition to periodic upsurges [4]. Moreover, the prevalence of emm types has been shown to exhibit temporal and geographical variations $[5,6]$. Several factors are thought to play a role in disease fluctuations, including prominent circulating emm types, increased host susceptibility, environmental factors and seasonal viral infections such as influenza [4-9].

iGAS has been notifiable in Ireland since 2004 under the Infectious Diseases Regulations 1981 as amended by S.I. no 707 of 2003. Cases are classified as confirmed, probable or possible, based on nationallyagreed case definitions, combining laboratory and clinical criteria [10]. Since 2005, enhanced surveillance, which includes collection of information on isolate site, clinical presentation, risk factors and patient outcome, has been conducted for iGAS cases on a voluntary basis [11]. In 2012 a laboratory reference service commenced to which isolates are voluntarily submitted for emm sequence typing.

\section{Methods}

\section{Clinical data collection}

Demographic information on iGAS cases was analysed using data which had been entered in the national electronic infectious disease reporting system (Computerised Infectious Diseases Reporting System, CIDR). CIDR allows exchange of information between laboratories, regional departments of public health and the Health Protection Surveillance Centre (HPSC) and is used to notify iGAS and record enhanced surveillance findings.

\section{Epidemiological typing}

From early 2012 onwards, all available iGAS isolates ( $n=109$ in 2012; $n=67$ in January to June 2013) were submitted by clinical microbiology laboratories to the GAS reference laboratory for emm sequence typing. Isolates which had been archived in $2011(n=28)$ were sent to be typed retrospectively. Typing was performed by sequencing the hypervariable region of the emm gene as previously described [12]. 


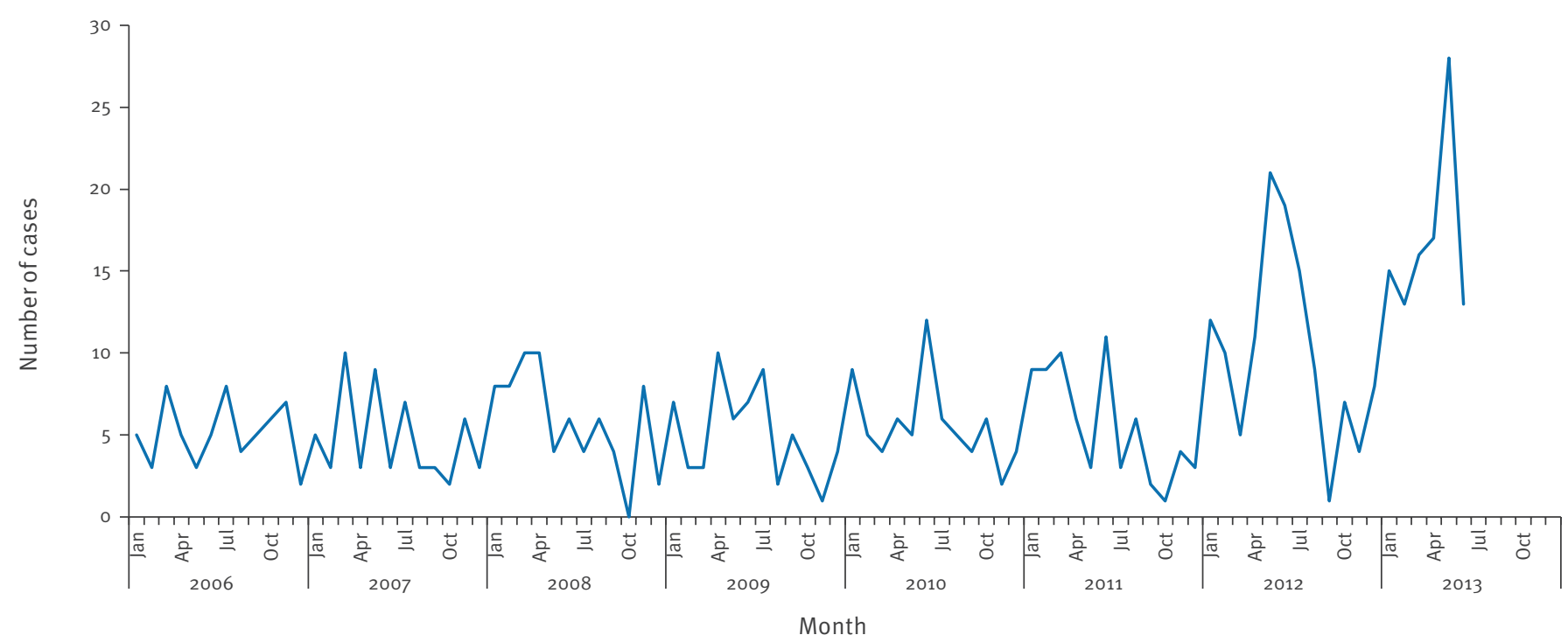

\section{Statistics}

Incidence rates were calculated using the 2011 population census data. Incidence rate ratios were calculated along with their 95\% confidence intervals ( $\mathrm{Cl}$; Fisher's exact test). The year 2005 was excluded from the analysis of enhanced surveillance data as there may not have been complete data reporting for that year. Difference in the emm type distribution was calculated using Fisher's exact test.

\section{Results}

\section{Epidemiology}

In 2012, 117 confirmed cases and five probable cases of iGAS infection were notified. This corresponds to an annual incidence of 2.66 cases per 100,000 (95\% Cl: 2.21-3.17 per 100,000). This is the highest annual incidence since iGAS became notifiable in 2004. Annual incidences for 2004 to 2011 have ranged from 0.8 per 100,000 in 2004 to 1.65 per 100,000 in 2008 , with incidence rates levelling out in 2009 to 2011 (range: 1.31-1.48 cases per 100,000) [11]. In 2012, the highest number of cases occurred in the months May through July, with the lowest in September (Figure 1). A further increase occurred during the first six months of 2013 with a total of 102 cases notified from January to June compared to 78 and 44 cases in the first six months of 2012 and 2011, respectively (Figure 1). The number of iGAS cases in May $2013(n=28)$ represents the highest number of notified cases in any month since iGAS became notifiable in 2004 .

In 2012, the age-specific incidence of invasive infection increased across most age groups when compared with the average age-specific incidence over the period 2006 to 2011 (Figure 2). The biggest increases and the highest number of cases occurred in older adults (older than 75 years) followed by children (under the age of four years; $p=0.053$ and $p=0.036$, respectively) (Figure 2). Males and females were represented equally in most age groups with the exception of those in the age range $0-9$ years where boys were more affected ( 8 female:18 male) and those in the age range 30-44 years where women were more affected ( 21 female:7 male). Overall, slightly more females than males were affected in 2012 (63 compared to 59); however, this was not statistically significant.

There were 26 cases of STSS in 2012 compared to an average of 6.17 cases per year for the period 2006 to 2011 (range: $3-9$ cases per year). The proportion of all iGAS cases that presented with STSS increased from $10 \%$ ( 37 of 383 ) for the period 2006 to 2011 to $21 \%$ in $2012(p=0.04)$. During the first half of 2013 , there were 22 cases of STSS accounting for $22 \%$ of all iGAS cases, indicating that the increased severity of iGAS infections observed in 2012 is sustained thus far in 2013.

In 2012 , eight patients died $(12.5 \%$ case fatality rate (CFR)) within seven days of onset of the disease for which GAS was identified as the main or contributing cause of death. Of these, six patients presented with STSS. There were five deaths (11.6\% CFR) in 2011, of which one case was associated with STSS, and an average of approximately three deaths per year ( $11 \%$ CFR) for the period 2006 to 2011, with approximately one case per year associated with STSS. For the first half of 2013 , there have been nine deaths (15.3\% CFR) with five cases associated with STSS.

\section{Typing results}

A total of 109 isolates (89\%) collected from reported iGAS cases in 2012 were typed by emm sequence typing (Figure 3). The most prominent types were emm1, 
Age-specific incidence rate of invasive group A streptococcal infections per 100,000 population by five-year age groups, Ireland, 2012 and averaged over the period 2006-11

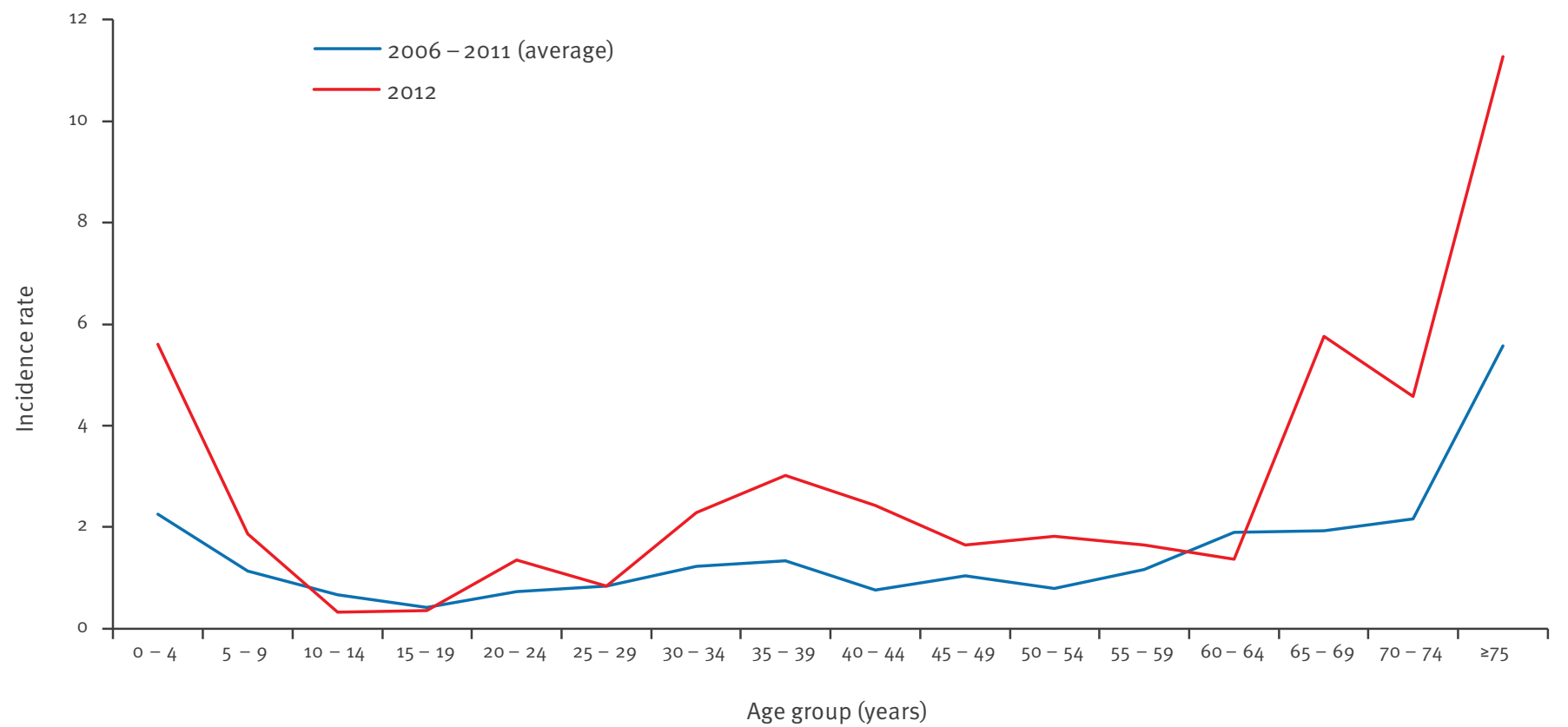

which accounted for $48.6 \%$ of isolates, followed by emm12 (9.2\%) and emm28 (7.3\%). Whereas, emm1 was equally distributed between males and females, emm12 and emm 28 were more predominant in males $(65 \%)$ and females (87.5\%), respectively, although neither value was significant ( $p=0.34$ and 0.065 , respectively). The emm1 type accounted for 17 of 25 STSS cases that were typed and for seven of eight deaths. Only two isolates of emm1 were detected from October to December 2012, when no cases of STSS were reported (Figure 3). Sixty-seven isolates (66\%) have so far been typed from all iGAS cases reported in January to June 2013. The number of emm1 isolates has declined somewhat, accounting for $40 \%$ of typed isolates compared to $54 \%$ of typed isolates for the same period in 2012 (JanuaryJune). However, there has been a notable increase in emm 3 in the first half of 2013 with $22 \%(n=15)$ of typed isolates belonging to this emm type contrasting with $1.5 \%(n=1)$ for the same period in $2012(p=0.0001)$ and $4 \%(n=4)$ in total for 2012 . There is limited information regarding the type distribution of iGAS in Ireland prior to 2012 . However, 28 iGAS isolates ( $42 \%$ of total) from 2011 were retrospectively typed and showed a lower prevalence of emm1 (25\%; eight of 28 ) and a higher prevalence of emm12 (32\%; nine of 28 ) compared to 2012 and 2013.

\section{Discussion}

A marked increase in notifications of iGAS occurred in Ireland in 2012 with a further increase observed in 2013. Increased incidences of iGAS have also been reported in 2012/13 in Finland, Norway, Sweden, and England, suggesting an increase in iGAS infections in Northern Europe [13,14]. Public Health England reported 1,038
iGAS cases for weeks $37 / 2012$ to $16 / 2013$ compared to 691 for $2011 / 12$ and 907 for $2008 / 09$, the last peak season for iGAS infection [14], indicating that the upsurge in Ireland (April-July 2012) pre-dated that in neighbouring England. iGAS has been notifiable in Ireland since 2004. It is therefore unlikely that the initiation of iGAS typing in 2012 and alerts issued on iGAS in July 2012 and April 2013 would have had a significant effect on the overall notification rate. There is evidence to suggest that viral infections such as influenza can contribute to an increased risk of invasive bacterial infections such as iGAS $[8,9]$. The short and mild 2011/12 influenza season in Ireland is unlikely to have had an impact on the increased incidence of iGAS in 2012. However, in $\mathbf{2 0 1 3}$, the influenza season was more prolonged and may have been a contributing factor to increased rates of iGAS [15].

The high prevalence of emm1 is likely to have played a significant role in the increased incidence of iGAS in 2012. The lower prevalence of emm1 and higher prevalence of emm12 in 2011 compared to the incidence of these types in 2012 suggests a possible change in the epidemiology of GAS between 2011 and 2012. However, no major conclusions can be drawn due to the small number of isolates which were typed in 2011. Significantly, England, Norway and Sweden have also reported a high prevalence of emm1 in 2012/13 [13,14]. The increased incidence of emm3 in 2013 is of concern given its association with a higher case fatality rate compared to other emm types [16]. Significantly, in 2013, emm3 accounted for five of eight fatal cases in Ireland for which typing data was available. An increased prevalence (11\%) of emm3 was also reported 
Quarterly reported invasive group A streptococcal cases $(n=224)$, proportion of typed isolates $(n=176)$ and most prevalent emm types, Ireland, January 2012-June 2013

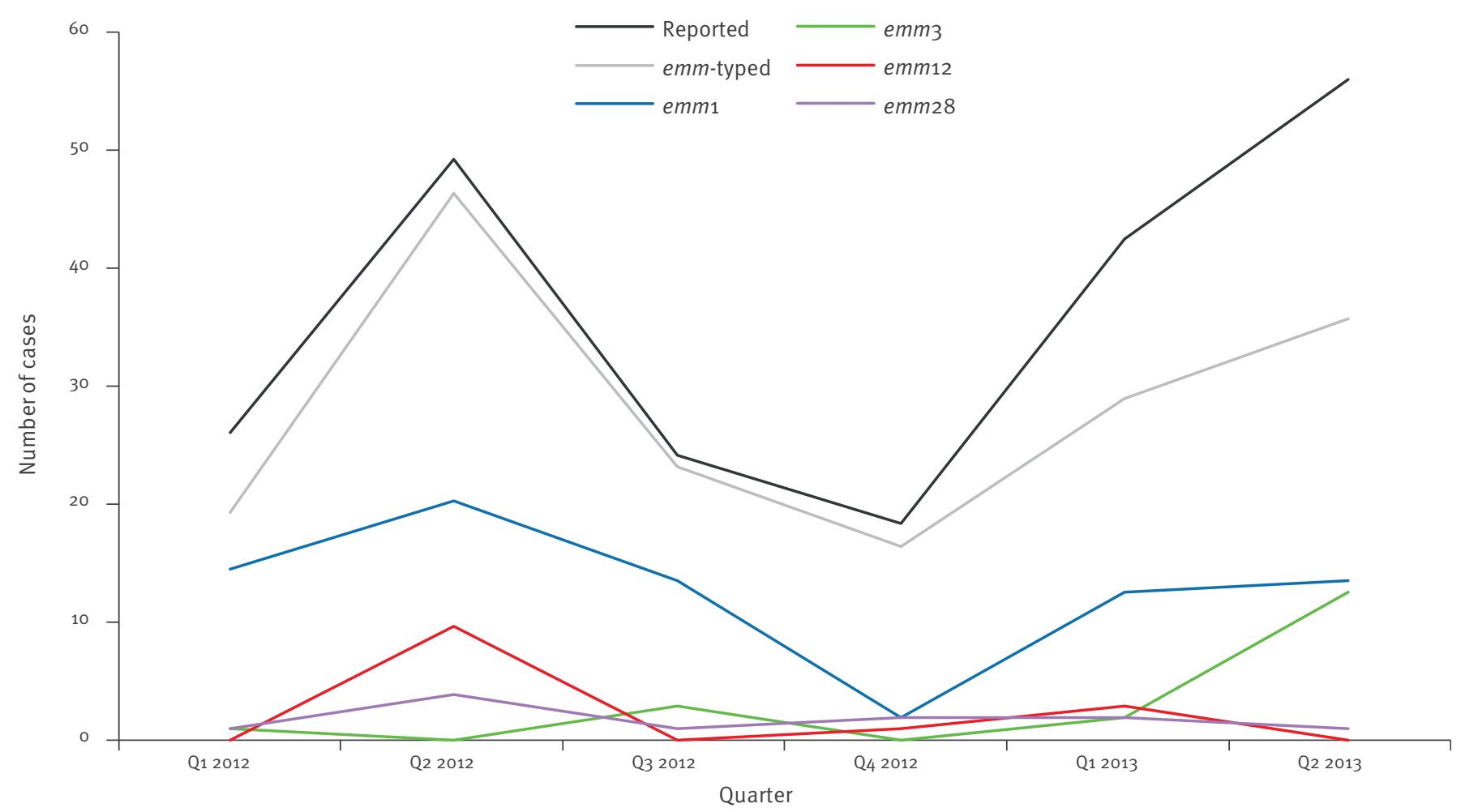

in Sweden in 2012 [13]. Continued typing of isolates will be required to confirm this trend in 2013 and to monitor any further changes in epidemiology.

The incidence of iGAS infections in Ireland, although increased in 2012, was lower than the 2012 upsurge rate reported in Sweden (6.1 per 100,000 population) [13]. Moreover, the overall annual incidence rates (0.81.65 in 2004-11) in Ireland prior to the upsurge were lower than that reported in the United Kingdom (UK), the Nordic countries and North America, which have in general higher reported incidence rates in the range 2.5-5.0 per $100,000[4,11,13]$. The reason(s) for the lower incidence rate of iGAS in Ireland is unclear [11]. A relatively high empiric antibiotic consumption has previously been suggested as a likely explanation [11]. For example, in 2010, outpatient penicillin use was higher in Ireland at 10.70 defined daily doses (DDD) per 1,000 inhabitants per day than in Finland at 6.6 DDD, Sweden at 7.1 DDD and the UK at 8.6 DDD per 1,000 inhabitants per day [17]. A small number of cases may also have gone unreported due to differences in case definitions between Ireland and other countries as previously reviewed [11]. Temporal and geographical fluctuations in iGAS infections may also be due in part to emm type distribution, population susceptibility, prevalence of certain risk factors, and environmental factors such as climate and population density $[4,11,18]$.
Similar to other European countries, seasonal variation in iGAS infections was observed in Ireland, with lowest incidence rates in late summer/autumn as reported elsewhere $[4,13,19]$. The peak rate of iGAS infections in Ireland in May 2012 and 2013 occurred later than in Sweden and other Nordic countries which generally exhibit peak incidences in the first quarter of each year $[13,19]$. However, occasional peaks in summer can also occur in these countries $[13,19]$. Noteworthy is the rising incidence of iGAS infections reported in April 2013 in England, possibly indicating a late upsurge similar to that observed in Ireland [14]. Seasonal variations in iGAS may reflect climatic differences such as daylight/ sunlight hours, rainfall/humidity, temperatures and seasonal behavioural patterns $[4,19]$. Certainly, spring 2012 and 2013 exhibited below average temperatures throughout Ireland.

Given the severity and rapid progression of iGAS, prompt detection and medical intervention are the only preventative and control measures available to reduce morbidity and mortality. In response to the increase in iGAS notifications, alerts and guidance have been issued to public health departments and clinicians (particularly general practitioners, emergency physicians, paediatricians, infection specialists and intensive care specialists), and iGAS recognition and management have been highlighted in surveillance bulletins. Laboratories are encouraged to send iGAS 
isolates for typing and to submit enhanced surveillance data to monitor the continuing trends in iGAS epidemiology, to investigate any suspected outbreaks and improve our understanding of iGAS epidemiology in Ireland. Moreover, in light of the reported increase in iGAS in several other European countries and the changing epidemiology of iGAS detected here in Ireland, public health institutions in Europe should be alert to any changes in epidemiology for the forthcoming 2013/14 iGAS season. Consideration should also be given to expanding the European Invasive Bacterial Disease Surveillance Network (EU-IBD) to include iGAS.

\section{Acknowledgements}

The authors would like to thank microbiology laboratories and departments of public health for reporting iGAS cases and submission of enhanced surveillance data. Special thanks also to microbiology laboratories for sending iGAS isolates for typing.

\section{Conflict of interest}

None declared.

\section{Authors' contributions}

Mary Meehan and Stephen Murchan performed the analyses and wrote the manuscript. Mary Meehan had overall responsibility for epidemiological typing and collating iGAS isolate data. Stephen Murchan had overall responsibility for collating enhanced clinical data of iGAS cases. Sarah Bergin provided clinical advice. Robert Cunney is the clinical lead for iGAS surveillance at the Health Protection Surveillance Centre, and director of Epidemiology and Molecular Biology Unit and, Irish Meningococcal and Meningitis Reference Laboratory. Darina O'Flanagan is director of the Health Protection Surveillance Centre. All authors supported the analyses, critically read the manuscript and approved the final submitted draft.

\section{References}

1. Steer AC, Lamagni T, Curtis N, Carapetis JR. Invasive group A streptococcal disease: epidemiology, pathogenesis and management. Drugs. 2012; 72(9):1213-27. http://dx.doi. org/10.2165/11634180-000000000-00000. PMid:22686614.

2. Centres for Disease Control and Prevention (CDC). Streptococcus pyogenes emm sequence database. Atlanta: CDC. [Accessed 14 Aug 2013]. Available from: http://www.cdc. gov/ncidod/biotech/strep/types_emm103-124.htm

3. Lynskey NN, Lawrenson RA, Sriskanden S. New understandings in Streptococcus pyogenes. Curr Opin Infect Dis. 2011;24(3):196-202. http://dx.doi.org/10.1097/ QCO.obo13e3283458f7e. PMid:21415743.

4. Lamagni, TL, Darenberg J, Luca-Harari B, Siljander T, Efstratiou A, Henriques-Normark B, et al. Epidemiology of severe Streptococcus pyogenes disease in Europe. J Clin Microbiol. 2008;46(7):2359-67. http://dx.doi.org/10.1128/JCM.00422-08. PMid:18463210. PMCid:PMC2446932.

5. Luca-Harari B, Darenberg J, Neal S, Siljander T, Strakova L, Tanna A, et al. Clinical and Microbiological Characteristics of severe Streptococcus pyogenes disease in Europe. J Clin Microbiol. 2009;47(4):1155-65. http://dx.doi.org/10.1128/ JCM.02155-08. PMid:19158266. PMCid:PMC 2668334

6. O’Brien KL, Beall B, Barrett NL, Cieslak PR, Reingold A, Farley $M M$, et al. Epidemiology of invasive group A streptococcus disease in the United States, 1995-1999. Clin Infect Dis. 2002;35(3):268-76. http://dx.doi.org/10.1086/341409. PMid:12115092.
7. O'Loughlin RE, Roberson A, Cieslak PR, Lynfield R, Gershman $\mathrm{K}$, Craig A, et al. The epidemiology of invasive group $A$ streptococcal infection and potential vaccine implications: United States, 2000-2004. Clin Infect Dis. 2007;45(7):853-62. http://dx.doi.org/10.1086/521264. PMid:17806049.

8. Scaber J, Saeed S, Ihekweazu C, Efstratiou A, McCarty N, O'Moore E. Group A streptococcal infections during the seasonal influenza outbreak 2010/2011 in South East England. Euro Surveill. 2011;16(5):pii=19780. Available from: http:// www.eurosurveillance.org/ViewArticle.aspx?Articleld $=19780$. PMid:21315058.

9. Zakikhany K, Degail MA, Lamagni T, Waight P, Guy R, Zhao $\mathrm{H}$, et al. Increase in invasive Streptococcus pyogenes and Streptococcus pneumoniae infections in England, December 2010 to January 2011. Euro Surveill. 2011;16(5): pii=19785. Available from: http://www.eurosurveillance.org/ViewArticle. aspx?Articleld=19785. PMid:21315057.

10. Health Protection Surveillance Centre (HPSC). Case definitions for notifiable diseases 2012 version 1.1. Dublin: HPSC; 2012 Available from: http://www.hpsc.ie/hpsc/NotifiableDiseases/ CaseDefinitions/File,823,en.pdf

11. Martin J, Murchan S, O'Flanagan D, Fitzpatrick F. Invasive Group A streptococcal disease in Ireland, 2004-2010. Euro Surveill. 2011;16(41): pii=19988. Available from: http://www. eurosurveillance.org/ViewArticle.aspx?Articleld=19988. PMid:22008200.

12. Beall B, Facklam R, Hoenes T, Schwartz B. Survey of emm gene sequences and T-antigen types from systemic Streptococcus pyogenes infection isolates collected in San Francisco, California; Atlanta, Georgia; and Connecticut in 1994 and 1995. J Clin Microbiol. 1997;35(5):1231-35. PMid:9114412. PMCid:PMC 232734.

13. Darenberg J, Henriques-Normark, B, Lepp T, Tegmark-Wisell $\mathrm{K}$, Tegnel A, Widgren K. Increased incidence of invasive group A streptococcal infections in Sweden, January 2012-Febuary 2013. Euro Surveill. 2013;18(14): pii=20443. Available from: http://www.eurosurveillance.org/ViewArticle. aspx?Articleld=20443. PMid:23594518.

14. Public Health England (PHE). Group A streptococcal infections: update on seasonal activity 2012/2013. Health Protection Report. 2013;7(17). Available from: http://www.hpa.org.uk/hpr/ archives/2013/news1713.htm

15. Health Protection Surveillance Centre (HPSC). Influenza surveillance in Ireland-weekly report: Influenza week 20 2013 (13th-19th May 2013). Dublin: HPSC; 2013. Available from: http://www.hpsc.ie/hpsc/A-Z/Respiratory/Influenza/ Seasonallnfluenza/Surveillance/InfluenzaSurveillanceReports/ 20122013Season/File,14095,en.pdf

16. Meisal R, Hoiby EA, Aaberge IS, Caugant DA. Sequence type and emm type diversity in Streptococcus pyogenes isolates causing invasive disease in Norway between 1998 and 2003. I Clin Microbiol. 2008;46(6):2102-05. http://dx.doi.org/10.1128/ JCM.00363-08. PMid:18417661. PMCid:PMC2446838.

17. European Centre for Disease Prevention and Control (ECDC). Surveillance of antimicrobial consumption in Europe, 2010. Stockholm: ECDC; 2013. Available from: http://ecdc.europa. eu/en/publications/Publications/antimicrobial-antibioticconsumption-ESAC-report-2010-data.pdf

18. Factor SH, Levine OS, Schwartz B, Harrison LH, Farley MM, McGreer A, et al. Invasive group A streptococcal disease: risk factors for adults. Emerg Infect Dis. 2003;9(8):970-7. http://dx.doi.org/10.3201/eido908.020745. PMid:12967496. PMCid:PMC3020599.

19. Siljander T, Lyytikäinen O, Vähäkuopus S, Snellman M, Jalava J, Vuopio J. Epidemiology, outcome and emm types of invasive group A streptococcal infections in Finland. Eur J Clin Microbiol Infect Dis. 2010;29(10):1229-35. http://dx.doi.org/10.1007/ s10096-010-0989-9. PMid:20563620. 